that anastrozole was better tolerated, produced fewer adverse events, had a superior riskbenefit profile and was associated with a lower breast cancer recurrence rate than tamoxifen.

Study participants were postmenopausal women with early-stage breast cancer, who received anastrozole $(n=3,125)$ or tamoxifen $(n=3,116)$. Compared with tamoxifen, anastrozole was associated with fewer treatmentrelated adverse events, fewer adverse events leading to withdrawal, and fewer serious adverse events. In individuals who received anastrozole, there was a reduced incidence of endometrial cancer, and fewer cerebrovascular and thromboembolic events, gynecologic events, and urinary infections were noted. Bone fractures, however, were more frequent in the anastrozole group, in which there was an increased incidence of osteopenia and osteoporosis. Vaginal dryness and dyspareunia were also more common in the anastrozole group. Risk-benefit analysis for anastrozole versus tamoxifen produced a hazard ratio of 0.85 on the Global Index of the Women's Health Initiative, and a ratio of 0.88 on the Global Index of Disease-Free Survival and Serious Adverse Events. The benefit from anastrozole was greatest at 1-2 years of treatment. The authors conclude that these data support the use of anastrozole as the initial adjuvant treatment for postmenopausal women with early-stage breast cancer.

Original article Buzdar A et al.; The Arimidex, Tamoxifen, Alone or in Combination (ATAC) Trialists' Group (2006) Comprehensive side-effect profile of anastrozole and tamoxifen as adjuvant treatment for early-stage breast cancer: long-term safety analysis of the ATAC trial. Lancet Oncol 7: 633-643

\section{Comparison of fluorouracil regimens after surgery for rectal cancer}

Postoperative radiotherapy combined with 5-fluorouracil (5-FU) treatment improves outcome in rectal cancer. Some studies have suggested superiority for protracted venous infusion (PVI) of 5-FU over bolus administration; others have found improved outcome associated with biochemically modulated 5-FU treatment compared with 5-FU alone. Smalley et al. therefore conducted a randomized phase III trial of 1,917 patients, comparing three chemoradiotherapy regimens: bolus 5-FU before and after radiotherapy combined with PVI 5-FU during radiotherapy (arm 1); 5-FU administered by PVI before, during and after radiotherapy (arm 2); and bolus 5-FU with leucovorin and levamisole before and after radiotherapy, with bolus 5-FU with leucovorin during radiotherapy (arm 3).

No difference was found in overall or diseasefree survival between the three arms; 3-year overall and disease-free survival rates ranged from $81 \%$ to $83 \%$ and from $67 \%$ to $69 \%$, respectively. Locoregional failure ranged from $4.6 \%$ to $8 \%$. Grade 3-4 hematologic toxicity was substantially higher in the bolus arms (55\% for arm 1 and $49 \%$ for arm 3) than in the PVIonly arm (4\%), and was higher in women than in men. Catheter-related toxicity was low. Fifteen patients died as a result of treatment, but lethal toxicity did not differ between arms.

The authors conclude that the methods of 5-FU administration examined in the study produce equivalent outcomes, although the toxicity profile varied between arms. The low relapse rate supports the use of initial tumor resection for selected patients.

Original article Smalley SR et al. (2006) Phase III trial of fluorouracil-based chemotherapy regimens plus radiotherapy in postoperative adjuvant rectal cancer: GI INT 0144. J Clin Oncol 24:3542-3547

\section{Comparing LTA with TH for the treatment of gastric cancer}

Gastric cancer of the cardia or subcardia is often treated with a left thoracoabdominal approach (LTA). Prognosis is often poor even with this extensive procedure, however, and some researchers have therefore recommended use of a less-extensive abdominaltranshiatal approach (TH). Sasako et al. have recently reported results from a randomized, phase III study in Japan that compared the two approaches.

Between July 1995 and December 2003, 167 patients with Siewert type II-III adenocarcinoma of the gastric body or cardia with esophageal invasion of $\leq 3 \mathrm{~cm}$ were randomized to either TH $(n=82)$ or LTA $(n=85)$. The first interim analysis was performed for 146 of the 165 patients enrolled before October 2003 , and showed that median overall survival was higher for patients receiving $\mathrm{TH}$ than for 